\title{
Anabases
}

ANABASES Traditions et réceptions de l'Antiquité

6 | 2007

Varia

\section{Couple modèle ou couple maudit ? Hector et Andromaque dans l'Iliade de Joseph d'Exeter}

\section{Francine Mora}

\section{(2) OpenEdition}

1 Journals

Édition électronique

URL : http://journals.openedition.org/anabases/3333

DOI : 10.4000/anabases.3333

ISSN : 2256-9421

Éditeur

E.R.A.S.M.E.

Édition imprimée

Date de publication : 1 octobre 2007

Pagination : 101-112

ISSN : 1774-4296

\section{Référence électronique}

Francine Mora, «Couple modèle ou couple maudit? Hector et Andromaque dans I'lliade de Joseph d'Exeter », Anabases [En ligne], 6 | 2007, mis en ligne le 01 janvier 2012, consulté le 20 octobre 2019 URL : http://journals.openedition.org/anabases/3333 ; DOI : 10.4000/anabases.3333

(c) Anabases 
Anabases 6 (2007), p. 101-112.

\section{Couple modèle ou couple maudit ? Hector et Andromaque dans l'Iliade de Joseph d'Exeter}

FRANCINE MORA

«Oh ! non, Hélène ! [...] Penser que nous allons souffrir, mourir, pour un couple officiel, que la splendeur ou le malheur des âges [...] vont se fonder sur l'aventure de deux êtres qui ne s'aimaient pas, c'est là l'horreur [...]. - Pourquoi ? S'il suffit d'un couple parfait pour vous faire admettre la guerre, il y a toujours le vôtre, Andromaque." (Jean Giraudoux, La Guerre de Troie n'aura pas lieu, Acte II, scène VIII)

LE XII SIÈCLE, QUI A VU NATTTRE LE GENRE ROMANESQUE à partir des « mises en roman » ou romans d'antiquité qui s'étaient fixé comme tâche de traduire ou plutôt d'adapter librement en " roman ", c'est-à-dire en français, de grandes épopées latines comme l'Énéide ou la Thébaïde, a aussi donné naissance à des épopées médio-latines de facture très classique souvent postérieures (et non antérieures, comme on aurait pu le penser) aux « mises en roman ». L'Iliade de Joseph d'Exeter est l'une d'entre elles ${ }^{1}$. Rédigée entre 1183 et 1190 par un clerc anglais, neveu de l'archevêque Baudouin de Canterbury auquel elle est dédiée, elle raconte sur quelques milliers d'hexamètres dactyliques répartis en six livres la guerre de Troie non pas d'après Homère, auquel les clercs médiévaux, faute de savoir le grec, n'avaient pas accès, mais d'après la De excidio Troiae historia de Darès le Phrygien, une très sèche mise en prose d'époque tardive (on la date du VIe siècle environ) qui a passé pendant tout le Moyen Âge, et même au-delà, pour un modèle

1 Joseph d'Exeter, L'Iliade, épopée du XIIe siècle sur la guerre de Troie, éd. L. Gompf, trad. et notes sous la direction de F. Mora, introduction de J. Y. Tilliette, Turnhout, Brepols, coll. "Miroir du Moyen Âge ", 2003. Toutes nos citations à venir proviendront de cette édition. 
d'exactitude historique et qui constitue aussi la source principale du Roman de Troie composé vers 1165 par Benoît de Sainte-Maure, sans doute pour la cour de Henri II Plantagenêt, roi d'Angleterre. Comme j'ai déjà tenté de le montrer ailleurs ${ }^{2}$, on peut soupçonner l'existence d'une rivalité poétique sous-jacente entre le roman français et l'épopée latine dans la mesure où les deux œuvres traitent du même sujet, sont issues de deux milieux à la fois proches et antagonistes - la cour de Henri II et l'archevêché de Canterbury, dont le titulaire avait été, peu de temps avant Baudouin, Thomas Becket et enfin s'opposent tant par la langue choisie que par le public potentiellement visé - d'un côté les clercs, de l'autre les chevaliers -, même si Joseph ne fait jamais la moindre allusion à Benoît, son prédécesseur, sans doute parce qu'il était inconcevable, vu les canons poétiques du temps, qu'un clerc latinisant avoue avoir lu et médité une ouvre écrite en vulgaire "roman ". L'écart idéologique entre les deux textes apparaît particulièrement bien dans le traitement profondément divergent qui est fait du couple vedette constitué par Hélène et Pâris : modèle d'amour partagé et de courtoisie chez Benoît, il devient chez Joseph un exemple accompli de perversité, Pâris, le traitre adultère, étant expédié après sa mort au fin fond du Tartare et Hélène, la femme impudique dominée par une sensualité dévorante, ne survivant que pour illustrer le triomphe du mal chez les humains ${ }^{3}$. On peut donc se demander si une divergence analogue n'apparait pas dans le traitement d'autres personnages et notamment d'un autre couple, celui d'Hector et d'Andromaque, lui aussi "emblématique de Troie » et à ce titre destiné en principe, ce qui se vérifie dans la pièce de Giraudoux, à contrebalancer celui d'Hélène et de Pâris. Catherine Croizy-Naquet a en effet bien montré que dans le Roman de Troie, face au modèle courtois incarné par les deux amants, Hector compose une figure ambiguë tandis qu'Andromaque ne joue qu'un rôle très effacé 4 . À l'inverse, on pourrait donc s'attendre à trouver dans l'Iliade de Joseph, face à un couple d'amants présenté comme diabolique, un couple conjugal exemplaire. Telle est bien en effet l'impression qui semble d'abord se dégager à première lecture, à travers l'héroïsation d'Hector et l'amour que lui voue Andromaque. Mais deux éléments originaux propres à Joseph, la comparaison d'Hector avec Henri III le Jeune Roi, fils aîné de Henri II, et une description détaillée autant qu'horrifique du songe prémonitoire qui annonce à Andromaque la mort d'Hector, amènent finalement à revenir sur cette première impression et à déceler dans l'Iliade, comme dans Troie, un certain nombre de réserves qu'il faudrait mettre en

2 F. Mora, "L'Ylias de Joseph d'Exeter : une réaction cléricale au Roman de Troie de Benoît de Sainte-Maure", in Progrès, réaction, décadence dans l'Occident médiéval, E. Baumgartner et L. Harf-Lancner (éd.), Genève, Droz, 2003, p. 199-213. Description, rewriting and authorship from Macrobius to medieval romance, Leiden, Brill, 1999, ch. IV (p. 121-170). Lui ne pense pas toutefois que Joseph ait connu Benoit.

4 C. Croizy-Naquet, "Le portrait d'Hector dans le Roman de Troie de Benoît de SainteMaure", Bien dire et bien aprandre 14 (1996), p. 63-77. C'est à elle que nous avons emprunté (p. 69) la formule qui précède. 
relation, pensons-nous, avec les difficultés que rencontrait la société du XIIe siècle à cause de la violence de ceux qu'on appelait alors les juvenes, les « jeunes».

Puisque le De excidio Troiae de Darès est la source commune de Joseph et de Benoît, il faut d'abord se demander sous quel jour y apparaît le couple formé par Andromaque et Hector. D'abord brièvement décrits au milieu d'autres Troyens dans la galerie de portraits qui constitue l'une des originalités du De excidio, ils jouent ensuite un rôle important, voire prépondérant, dans le passage narratif qui décrit le début de la guerre, du débarquement des Grecs à la mort d'Hector ${ }^{5}$. L'élément dominant du couple, mais aussi le plus ambigu, est Hector, ce qui se manifeste dès la galerie de portraits. Alors qu'Andromaque est dépeinte de manière essentiellement mais banalement méliorative, puisqu'elle est dite "resplendissante (candidam), élancée, jolie, modeste, avisée, pudique, agréable ", dans une brève évocation qui mêle avantages physiques et qualités morales ${ }^{6}$, Hector bénéficie d'un portrait plus long et surtout beaucoup plus original, car plus équivoque. Lui aussi est " resplendissant " (candidum), mais chez lui cette splendeur est altérée par deux défauts physiques majeurs (il est bègue et il louche, blaesum [...] strabum), contradiction interne qui se retrouve dans son portrait moral, puisqu'il est dit à la fois "belliqueux » et "clément " (bellicosum [...] clementem). Sa description semble ainsi bâtie sur le mode de l'oxymore, ce qui la rend assez déconcertante ${ }^{7}$. Or cette ambiguïté foncière semble se retrouver dans le passage narratif où il est mis en vedette, dès le débarquement des Grecs et le début des combats, comme chef des armées troyennes : praefecit Priamus principem et ductorem Hectorem ${ }^{8}$. Ce passage donne en effet l'impression d'avoir bénéficié d'une relative élaboration stylistique, ce qui est rare chez Darès, dont la prose minimale constitue souvent le degré zéro de la poétique, ce qui n'a d'ailleurs pas peu contribué à le faire passer pour un modèle d'objectivité historique. On y relève d'abord quelques emprunts au lexique virgilien comme l'emploi de Graiugenae pour désigner les Grecs, ou d'Orcus pour qualifier les Enfers, emprunts qui, si disséminés qu'ils soient, contribuent à donner au texte une coloration discrètement épique ${ }^{9}$. Deux autres procédés manifestement concertés viennent par ailleurs ponctuer régulièrement la narration et sont de nature à avoir pu

5 Daretis Phrygii de excidio Troiae historia, éd. F. MeISTER, Leipzig, Teubner, 1873, ch. XII (galerie de portraits) et XVIII-XXV (début des combats). L'Historia de Darès a été récemment traduite par G. FRY dans Récits inédits sur la guerre de Troie, Paris, Les Belles Lettres, 1998, p. 231-287.

6 Darès, De excidio, XII, 15-17 : Andromacham [...] candidam longam formosam modestam sapientem pudicam blandam.

7 Darès, XII, 1-4 : Hectorem blaesum candidum crispum strabum (etc) : « Hector était bègue, replendissant, avait les cheveux bouclés, louchait (etc.) ".

8 Darès, XVIII, 6-7 : "Priam plaça Hector comme généralissime et commandant en chef " (G. FRY, Récits, p. 263).

9 Darès, XX, 7 ; XXIV, 16 (Graiugenae) et XXI, 8 (Orco). G. Fry note ces emprunts, en renvoyant à chaque fois à Virgile (Énéide, III, 550 et II, 398). 
éveiller des échos dans la mémoire de lecteurs qui, bien que clercs, devaient garder le souvenir de quelques chansons de geste : d'une part des hyperboles numériques insistant soit sur la longueur des combats, soit sur l'ampleur des massacres, d'autre part des répétitions formulaires comme fit magna caedes, "le massacre est grand ", ou nox proelium dirimit, "la nuit met un terme aux combats ", qui évoquent curieusement à l'esprit, par exemple, certains vers d'intonation des laisses de la Chanson de Roland: « la bataille est merveilluse e pesant ${ }^{10}$ ». Dans ce passage privilégié, la narration de Darès prend donc des accents discrètement épiques, et c'est avant tout Hector qui en bénéficie. En tant que chef des armées, il est en effet mentionné le premier, et c'est à lui que sont attribuées les plus longues énumérations de victimes : "le massacre est grand. Hector tue l'Orchoménien Ialmenus, Épistrophe, Schédius, Éléphénor, Diorès, Polyxénus. Énée : Amphimaque et Nirée 11 ». Un processus d'héroïsation guerrière cherche visiblement à se mettre en place, renforcé par la présence d'Énée, qui joue ici le rôle de faire-valoir, ce que confirme d'ailleurs la suite du récit de Darès, après la mort d'Hector : ce sont les funérailles de ce dernier qui sont mentionnées, non plus celles de Patrocle - innovation majeure par rapport à Homère, comme le note Gérard Fry -, et c'est le souvenir glorieux du guerrier défunt qui sert de référence pour mesurer la vaillance des survivants ${ }^{12}$. En même temps, toutefois, la pauvreté répétitive du lexique de Darès martèle à l'envi des formules comme Hector occidit ou Hector interficit, ce qui, joint aux énumérations récurrentes de victimes, donne à voir avant tout le fils aîné de Priam comme une sorte de massacreur infatigable, ce qui souligne son efficacité mais limite quand même ses capacités ${ }^{13}$. Face à lui Andromaque apparaît d'ailleurs bien effacée, et bornée au registre du planctu femineo, de la plainte féminine ${ }^{14}$. La formule qui la définit, répétée deux fois, Andromacha maesta, "la triste Andromaque », confirme ce rôle qui lui est donné de pleureuse inefficace, impuissante à retenir Hector malgré son rêve prémonitoire, et de victime toute désignée des combats, dernière image que nous laisse d'elle le texte de Darès ${ }^{15}$.

Lévocation du couple formé par Hector et Andromaque n'est donc, on le voit, que très partiellement méliorative dans la De excidio Troiae historia, ce que semble bien avoir perçu Benoît, lecteur attentif du prosateur latin. Comme a pu le montrer

Darès, XX, 25 ; XXI, 8 ; XXII, 8 ; XXIII, 9 (fit magna caedes) ; XX, 1-2 ; XXI, 15-16 ; XXIV, 14 (nox proelium dirimit). Cf. La Chanson de Roland, éd. et trad. I. Short, Paris, Le Livre de Poche, coll. « Lettres Gothiques », 1990, laisse 104, v. 1320 ; laisse 110, v. 1412 ; laisse 123 , v. 1610 et laisse 124 , v. 1620.

Darès, XXI, 17-20 : fit magna caedes : Hector Orcomeneum Ialmenum Epistrophum Schedium Elephenorem Dioren Polyxenum duces occidit, Aeneas Amphimachum et Nireum.

Darès, XXIV, 2-3 (Andromacha maesta) ; XXIV, 8 (maesta Andromacha) et fin du ch. XLIII. 
Catherine Croizy-Naquet, Hector bénéficie certes dans le Roman de Troie d'un processus d'héroïsation qui se manifeste tant dans les éloges réitérés du narrateur que dans sa mise en valeur posthume à travers l'érection d'un tombeau magnifique dont la description est beaucoup plus développée que chez Darès, mais la prouesse de ce guerrier modèle apparaît trop souvent " pervertie par son excès ", un excès qui fait qu'il n'est " jamais loin de la cruauté ou d'une férocité primitive », ce que traduit stylistiquement sa comparaison fréquente avec des animaux sauvages. Le bégaiement d'Hector pourrait alors selon elle être interprété comme le signe d'un rapport à la parole inefficace car "perverti », le seul langage que sache manier ce guerrier étant en fait " celui des armes et de la force physique ". Face à lui Andromaque est d'ailleurs, conclut-elle, " très vite évincée ", les qualités que le narrateur lui prête au départ restant largement "inexploitées, voire ignorées 16 ». En va-t-il de même chez Joseph, lui qui avait plutôt intérêt, nous l'avons vu, à faire d'Hector et Andromaque un couple exemplaire, pour faire pendant au couple diabolique d'Hélène et de Pâris?

Dans l'Iliade de Joseph, les premières mentions d'Hector laissent assurément le lecteur un peu perplexe, car elles semblent aller, comme chez Darès, dans des sens différents, voire opposés. La toute première mention qui en est faite au livre II le qualifie en effet de "craintif " (pavidus Hector : "le craintif Hector ", v. 198), ce qui paraît a priori contradictoire avec l'unique allusion du livre suivant, le livre III, qui l'héroïse implicitement en le comparant à Hercule - une triple allitération venant encore renforcer le processus d'assimilation : par Herculis Hector (v. 92 : "Hector est l'égal d'Hercule »). L'impression d'ambiguïté peut être d'autant plus grande que dans le livre IV, qui reprend la galerie de portraits de Darès, les aspects paradoxaux du portrait d'Hector ne sont nullement gommés. Si la description est mieux organisée que dans le De excidio, elle n'en évolue pas moins de la mélioration à la péjoration en partant du « torse magnanime " du héros (magnanimum pectus, v. 49) pour aboutir, dans un très beau versus aureus, à cet œil louche qui, en perturbant sa vue, " assombrit d'une lumière mourante son ferme visage 17 ». Un peu comme le bégaiement dans le Roman de Troie, le strabisme se charge ici d'une valeur symbolique qui semble annoncer en filigrane la chute de Troie ${ }^{18}$. Il faut toutefois replacer ces éléments dans leur contexte. Ainsi la comparaison méliorative du livre III est un cri unanime du peuple troyen, tandis que l'épithète dépréciative du livre II est placée dans la bouche de Pâris, ce qui amène à reconsidérer sa portée et sa signification. Elle est en fait l'unique vestige, chez Joseph, d'un passage assez développé chez Darès, et conservé par Benoît : un discours en forme

16 C. Croizy-Naquet, "Le portrait d'Hector", p. 70-76.

17 Liliade, IV, 56 : occiduo stabilem contristat lumine vultum.

18 Voir notre article "Galerie de portraits et tombeau de Teuthras dans le livre IV du De Bello Trojano de Joseph d'Exeter : la perfection insaisissable”, PRIS-MA 16/ 2 (2000), p. 249-265. Voir aussi C. CROIZY-NAQUET, "Le portrait d'Hector", qui consacre un assez long développement (p. 72-75) au strabisme et au bégaiement. 
dans lequel Hector disait ses réserves à l'égard de toute expédition en Grèce, non sans raison, car cette expédition, finalement menée par Pâris, aboutit à l'enlèvement d'Hélène, et donc à la guerre de Troie. Joseph a sans doute supprimé ce passage parce qu'il jugeait que les tendances pacifistes qui s'y exprimaient n'étaient pas en accord avec la stature guerrière qu'il voulait donner à son héros. C'est pourquoi, usant avec finesse de la technique des points de vue, il a placé la très brève allusion qui y renvoie dans la bouche d'un personnage qu'il présente par ailleurs comme exécrable, voire démoniaque, ce qui fait apparaître l'accusation de lâcheté comme une simple calomnie et la vide de tout véritable poids. Si Pâris ironise sur les craintes supposées d'Hector, c'est en effet parce qu'il veut convaincre les Troyens de confier à lui, et à lui seul, la conduite de l'expédition en Grèce : «j'irai, j’irai! Ce n'est pas moi que vont terroriser les Syrtes dont le craintif Hector nous oppose les risques ${ }^{19}$. »

Même s'il a maintenu les paradoxes du portrait d'Hector, Joseph semble donc bien avoir travaillé à une homogénéisation de son héros, et plutôt dans le sens de la mélioration. Dans le livre V de l'Iliade, qui adapte les chapitres XVIII-XXIV de Darès, du début des combats à la mort d'Hector, le fils aîné de Priam incarne indéniablement la figure du guerrier exemplaire, non seulement héroïsé mais presque divinisé. Sa première réaction devant l'arrivée des Grecs mentionne certes sa peur (Hector [...] extimuit, v. 124-125), semblant faire écho au pavidus du livre II, mais très vite cette crainte initiale est surmontée par une " ardente bravoure " (ardens [...] virtus, v. 126-127) qui l'amène à se précipiter « seul " au devant de l'ennemi, l'adjectif solus étant bien mis en valeur par une reprise anaphorique ${ }^{20}$. Et ensuite cette bravoure ne se dément plus, comme en témoignent les épithètes très uniformément mélioratives qui accompagnent le nom du héros : premier (primus, v. 137) à passer à l'attaque, il est aussi le dernier (ultimus, v. 214) à battre en retraite, en "gard[ant] un air menaçant "; à chaque fois la métrique met en valeur ces épithètes, le v. 137 en s'ouvrant sur l'adjectif primus et en se fermant sur le nom d'Hector ${ }^{21}$, le v. 214 en plaçant la locution ultimus Hector dans sa clausule. Émule de Mars, dieu des combats, comme en témoignent les locutions Martius Hector (v. 340, v. 397) et Mavortius Hector (v. 525), elles aussi placées dans la clausule, le héros est même assimilé implicitement à Jupiter en personne par l'intermédiaire de l'adjectif fulmineus, "foudroyant ", appliqué tantôt à lui-même (fulmineus Hector, v. 300, mis en valeur par un rejet), tantôt à ses armes (hastam / fulmineam, v. 249, locution mise encore mieux en valeur par un rejet doublé d'un contre-rejet). Amorcée par ces épithètes métonymiques ou métaphoriques, l'entreprise d'héroïsation voire de divinisation finit même par devenir explicite vers la fin du livre V, d'abord

L'Iliade, II, 198-199 : ibo, ibo. Non me, pavidus quas obicit Hector/ terruerint sirtes. L'Iliade, V, 128-130 : rapit ilicet arma nec agmen / expectat solusque effracto cardine, solus/ evolat (" très vite il se jette sur ses armes et, sans attendre l'armée, c'est seul qu'il force la porte, seul qu'il vole au combat").

L'Iliade, V, 137 : primus ut Argolicos tonsis certantibus Hector. 
grâce à une comparaison en forme d'Hector avec Mars au moment où, malgré les supplications d'Andromaque, il repart au combat (" c'est avec moins d'ardeur qu'à travers les escarpements de l'Ossa, avec l'Effroi pour écuyer, Mars se rue 22 "), ensuite à travers le regret prononcé par le narrateur-poète sur le héros défunt : « il a péri, hélas, l'unique espoir des Phrygiens, le belliqueux Hector a péri ; lui qui, s'il avait en naissant reçu des membres immortels, se serait vu confier par Jupiter lui-même la tâche de projeter ses feux 23 ". Les allusions récurrentes au souvenir d'Hector qui parsèment le livre VI confirment du reste, encore mieux que chez Darès, l'exemplarité guerrière du personnage ; meilleur soutien de Troie (v. 25-26), toujours présent à la mémoire des Troyens qui croient le voir à chaque instant (v. 18), il reste la référence obligée de toute action héroïque, comme l'atteste par exemple la conclusion de l'éloge que le narrateur fait de Troillus, un autre fils de Priam : "J'enfermerai tous ses titres de gloire dans une brève formule, en le disant plus grand qu'Hector (Hectore maior) ${ }^{24}$."

Joseph s'est donc bien efforcé de donner à Hector une stature épique, et même héroïque au sens plein du mot, en suggérant de voir en lui une sorte de demi-dieu. Mais ce mouvement d'héroïsation était déjà en germe chez Darès, et se retrouve au moins en partie chez Benoît. Ce qui est plus surprenant, c'est que dans l'Iliade Andromaque elle aussi, plutôt terne, nous l'avons vu, dans le De excidio et dans Troie, se voit gratifiée d'une stature épique grâce au songe prémonitoire qui lui annonce la mort d'Hector. Ce songe est évoqué de manière minimale et vraiment plate chez Darès : "Lorsqu'arrive le moment de la bataille, Andromaque, la femme d'Hector, voit en songe qu'Hector ne doit pas s'engager dans la bataille ${ }^{25}$." Benoît fait un peu plus d'efforts de dramatisation en insistant sur le caractère fantastique de ce songe, signe de son origine surnaturelle : "Oiez cum fet demonstrement :/ icele nuit demeinement/ que la trive fu definee/ dut bien la dame estre esfree ;/ se fu ele, jel sai de veir./ Li deu li ont fet a saveir/ par signes e par visions/ e par interpretations/ son grant damage e sa dolor ${ }^{26}$. " Mais le contenu du rêve reste imprécis. Chez Joseph au contraire il est détaillé avec une précision remarquable, à travers une succession d'images à la fois terrifiantes et symboliques qui donnent corps aux « signes » et « visïons » de Benoît : «Voici que son profond repos fait naître, clairement figurés à l'image du réel, de terribles présages : adhérant solide-

L'Iliade, V, 466-467 : non sic ossea per ardua Mavors/ armigero Terrore ruit.

L'Iliade, V, 525-528 : occidit, heu, spes una Frigum, Mavortius Hector/ occidit; eternos cui si natura dedisset/ artus, ipse suos iaculandos Iupiter ignes/ [...] mandasset. Les références à Mars et à Jupiter, on le voit, s'entremêlent ici.

L'Iliade, VI, 191 : utque omnes claudam titulos brevis, Hectore maior. Cf. aussi les v. 687$688 \mathrm{du}$ livre VI, à propos d'Amphimaque, un autre frère d'Hector (on y retrouve la même clausule, Hectore maior).

Darès, De excidio, XXIV, 1-2 : at ubi tempus pugnae supervenit, Andromacha uxor Hectoris in somnis vidit Hectorem non debere in pugnam procedere.

Benoît de Sainte-Maure, Le Roman de Troie, éd.-trad. E. Baumgartner et F. Vielliard, Paris, Le Livre de Poche, coll. «Lettres Gothiques », 1998, v. 15279-15287. 
ment aux parois de la chambre, un laurier s'élevait, qui avait enlacé des dieux Lares inconnus ; une hache ennemie le coupe à la racine, ses rameaux, qui dégouttent de sang, tombent sur le lit nuptial. Son cauchemar affreux fait surgir à sa vue plusieurs autres prodiges : la voilà maintenant qui gît sans son époux et le corps répandu sur le lit tout entier ; elle voit des bouches ouvertes, ensanglantées, souiller encore une fois son sein nu ${ }^{27}$. "À travers le procédé des reprises allusives, deux modèles épiques sont convoqués ici : l'Énéide de Virgile et la Thébaïde de Stace, bien connus tous les deux des clercs du XII siècle. D'abord la clausule iunctissima laurus, associée à la locution amplexa lares (" un laurier s'élevait, qui avait enlacé des dieux Lares inconnus »), peut évoquer à l'esprit l'antique laurier (iuxtaque ueterrima laurus, autre clausule) qui, au livre II de l'Énéide, ombrage l'autel des Pénates dans le palais de Priam (complexa penatis) et assiste donc au meurtre du vieux roi par Pyrrhus 28 ; ensuite une nouvelle clausule, deux vers plus loin, rorantes sanguine rami, décrivant " les rameaux qui dégouttent de sang ", vient non sans habileté s'associer à la première tout en évoquant un célèbre songe épique, le songe qui, dans la Thébaïde, annonce à Atalante, mère de Parthénopée, la mort de son fils, en lui faisant voir " un chêne fendu par mille blessures, son feuillage à terre, ses branches mouillées de sang (rorantes sanguine ramos, toujours une clausule) et expirant sur le sol 29 \%. On voit comment, d'une clausule à l'autre, s'établit un faisceau de réminiscences qui coud l'un à l'autre deux passages épiques pour prédire la fin sanglante de la race de Priam, donnant à Andromaque, nouvelle Atalante ou nouvel Énée visité par les dieux, une stature hérö̈que : l'ensemble du songe est en effet introduit par la formule virgilienne nox erat, qui annonce toujours, dans l'Énéide, un songe d'Énée 30 .

La figure d'Andromaque prend donc dans l'Iliade un relief tout particulier. Est-ce à dire pour autant qu'elle forme avec Hector un couple parfait ? La réponse ne va pas de soi. Car il est vrai que dans un premier temps Joseph semble avoir voulu insister, plus que Darès et Benoît, sur la parfaite harmonie qui règne au sein du couple. En effet non

27 L'Iliade, V, 431-438: in veras matura quies purgata figuras/ dira movet: stabat thalamis iunctissima laurus/ secretos amplexa lares; inimica securis/ hanc a stirpe metit, rorantes sanguine ramil in stratum geniale cadunt. Plura ingerit horrens/ monstra sopor: iacet interdum sine coniuge totol fusa thoro, nudos rursus videt oris hiatus/ sanguineos fedare sinus. Virgile, Enéide, éd. et trad. J. Perret, Paris, Les Belles Lettres, 1981, II, 512-514 : ingens ara fuit iuxtaque ueterrima laurus/ incumbens arae atque umbra complexa penatis (" il y avait un autel colossal et auprès un laurier très vieux penché sur l'autel et de son ombre embrassant les Pénates "). On note que chez Virgile complexa penatis est aussi une clausule. Stace, Thébaïde, éd. et trad. R. Lesueur, Paris, Les Belles Lettres, 1994, IX, 595-597 : multo proscissam uulnere cernit/ deposuisse comam et rorantes sanguine ramos/ expirare solo. La clausule purgata figuras, chez Joseph, évoque de surcroît la clausule turbata figuris, placée à la fin du vers qui ouvre la description du songe d'Atalante (IX, 570 : tristibus [...] turbata figuris: "troublée par de sinistres visions"). 
seulement Andromaque est gratifiée, comme chez Darès, d'un portrait bref mais très mélioratif qui fait se succéder les substantifs élogieux (" la beauté, la mesure, la piété, l'intelligence, la pudeur rehaussent de leur parure Andromaque à la haute stature et à l'aspect serein $31 »)$, mais encore l'adjectif serenam qui clôt ce portrait se retrouve comme en écho dans le portrait d'Hector, loué pour son "visage serein " (faciem serenam ${ }^{32}$ ). Et la nuit où intervient le songe commence par s'ouvrir sur un tableau idyllique où l'étroite union des corps laisse présager celle des cœurs : "C'était pendant la nuit ; Andromaque gisait, étreignant son mari que la longue bataille avait exténué ; après avoir voulu par ses questions connaître les rigueurs du combat [...], assoupie elle repose, couchée sur son époux 33 . " On ne trouve rien de pareil ni chez Darès ni chez Benoît. Mais justement l'horreur du songe rompt cette union exemplaire. Aux cris entrecoupés et terrifiés d'Andromaque, "imaginant la perte du mari qu'elle étreint 34 ", Hector ne répond, comme chez Darès et Benoît, que par l'ardeur impitoyable d'un guerrier avant tout impatient de reprendre le combat : c'est alors qu'il est comparé au dieu Mars et qualifié, dans une clausule, de "féroce Hector" (ferus Hector, v. 471), une locution qui fait écho à une autre toute semblable intervenue plus haut au cœur des combats : efferus/ Hector, "le cruel Hector» (v. 404-405, avec un effet de rejet). Cette sauvagerie qui fait d'ailleurs d'Hector, tout au long du livre V, comme chez Darès, un massacreur sans égal, capable par exemple de tuer d'un seul coup trois guerriers ${ }^{35}$, rompt avec la sérénité notée plus haut et disjoint l'union d'un couple qui ne trouve plus son unité que dans la violence, violence du songe chez Andromaque, violence du combat chez Hector. C'est alors qu'on peut prêter attention à une autre innovation de Joseph, la comparaison qui est faite d'Hector, tout à la fin du livre V, juste après son trépas, avec Henri le Jeune Roi, fils aîné de Henri II : "Pareil en sa grandeur, il avait hardiment jusqu'à celles d'Hector fait enfler ses colères (excreverat iras), notre Henri le troisième [...] ; et la race de Mars, ayant trouvé dans la guerre son maître, ne lui contesta pas la faveur de Bellone, pas plus qu'on ne nous conteste celle qui vient de Pallas ${ }^{36}$. "

Bien que sa renommée ait ensuite été éclipsée par celle de son frère cadet Richard Cœur de Lion, cet Henri, très connu au XIIe siècle, a en effet laissé le souvenir d'un

31 L'Iliade, IV, 85-86 : forma, modus, pietas, sensus, pudor auctius ornant/ Andromachen erectam humeris visuque serenam.

32 L'Iliade, IV, 53 (serenam est là aussi mis en valeur dans la clausule).

33 L'Iliade, V, 425-427 et 429-430 : nox erat, et longo defessum Marte iacebat/ Andromache complexa virum; cumque aspera bellil quesisset, [...] sopita maritol incubat.

34 L'Iliade, V, 444 : amissumque putans complexa maritum.

35 L'Iliade, V, 306-307. Plus loin, dans le livre VI, Troïlus est d'ailleurs loué de « renouveler les massacres accomplis par son frère" (v. 195 : fraternas renovat cedes).

L'Iliade, V, 533-534 et 536-537 : tantus in Hectoreas audax excreverat iras/ tercius Henricus noster; [...]/ et in bellis gens Martia nacta priorem/ non illi invidit Bellonam ut Pallada nobis. 
curieux personnage. Joseph n'est pas le seul à le comparer à Hector : cette comparaison est reprise un peu plus tard par Gervais de Tilbury, qui l'avait bien connu, dans ses Otia imperialia, au sein d'un bref planctus en vers qui déplore, comme Joseph, la mort précoce du jeune roi : rosa forme singularis/ marcet, perit alter Paris/ Hector alter occubuit ${ }^{37}$. Il n'est pas certain toutefois que ce rapprochement soit uniquement mélioratif. Car Henri III a trouvé la mort au cours d'une rébellion qui l'avait dressé contre son père et qui l'avait amené à piller deux sanctuaires, ceux de Saint-Martial de Limoges et de Rocamadour, une action sacrilège qui ne pouvait recueillir l'approbation des clercs. C'est sans doute pourquoi Gautier Map, un familier de Henri II, livre de lui un long portrait très nuancé, et même oxymorique, où l'on retrouve les contradictions internes du portrait d'Hector. Certes Gautier reconnait la valeur guerrière du jeune roi («ce fut, écrit-il, un homme de guerre imaginatif, qui réveilla ce métier quasiment dormant et le conduisit à son apogée ") et célèbre bien volontiers "ses vertus et ses qualités ", mais, poursuit-il, « il utilisa toutes ces qualités pour le mal », ce qui nous vaut un remarquable défilé d'oxymores : "C'était [...] une source limpide de crimes, un agréable foyer de méchanceté, un splendide palais du péché »; bref, conclut-il, «il mourut furieux, mais le Seigneur ne l'était pas en regardant sa fin ${ }^{38}$. "Decessit iratus: on retrouve ici mention du sentiment ou du péché, ira, la colère, mis en exergue par Joseph dans sa comparaison d'Hector et d'Henri III, un péché qui, fait digne d'être noté, accompagne et cause aussi le décès du héros troyen ; c'est en effet parce qu'il a été blessé par Hector qu'Achille, devenu furieux (vulneris acrior ira), se jette sur son adversaire également furieux de ne pas l'avoir tué (pudor aggerat iram) et lui porte le coup mortel ${ }^{39}$. Un regard sans complaisance est ici porté par deux clercs sur la violence guerrière, une violence perçue essentiellement, l'exemple d'Henri III l'atteste, comme celle des « jeunes ». Marié à Marguerite de France et associé à la couronne du vivant de son père, mais tenu à l'écart du pouvoir par ce père encore très vert, Henri le Jeune Roi fournit en effet, comme le note Martin Aurell, l'image emblématique du "juvenis, fils de famille [...] qui, en attendant d'hériter, parcourt et pille en bande le pays 40 ». En témoigne le planh consacré à sa mémoire par le troubadour Bertran de Born, qui s'organise autour de la répétition du leitmotiv « le jove rei englés » et qui affirme qu’après sa mort, « reman Prètz e Jovens doloros » (« Mérite et Jeunesse restent affligés ») 41.

37 Gervais de Tilbury, Otia imperialia, éd. et trad. angl. de S.E. Banks et J.W. Binns, Oxford, Clarendon Press, 2002, II, 21, p. 486 (" une rose d'une beauté singulière s'est flétrie, un autre Pâris est mort, un autre Hector a péri »). p. 222-223 : fons scelerum serenissimus, appetibilis nequicie fomes, pulcherrima peccati regia (De nugis curialium, éd. M. R. James, Oxford, Clarendon Press, 1983, p. 282). L'Iliade, V, 500 et 502. 
Bien sûr, Hector, qui combat pour défendre sa patrie sous les ordres de son père, n'est pas exactement assimilable à un jeune rebelle. Il faut toutefois noter que dans son dernier combat il échappe au contrôle paternel, puisque Priam, répondant aux supplications d'Andromaque qui s'écrie : "Hélas, voici notre mort, père, secourez-nous !", veut l'empêcher de partir, mais que « c'est à peine si Hector, quand il en reçoit l'ordre, finit par s'arrêter devant le prince ", première velléité de révolte qui finit par déboucher sur l'accès de colère final qui entraîne sa mort ${ }^{42}$. Sa fougue irrépressible rejoint bien ici celle des " jeunes ». Un détail menu mais significatif semble d'ailleurs confirmer que Joseph voyait bien en lui, comme dans Henri III, un juvenis : Darès en effet le déclare barbu, barbatum, détail qui disparaît curieusement chez Benoît 43 ; Joseph, lui, maintient ce détail, mais transforme la barbe en un "premier duvet ", lanugo, en une barbe naissante qui, chez Virgile comme chez Juvénal, connote l'idée de jeunesse et qui dans l'Énéide caractérise même un combattant, Clytius, explicitement défini comme un tout jeune homme, un iuvenis ${ }^{44}$. Le songe d'Andromaque peut alors être relu à la lumière de cette assimilation, notamment dans sa dernière partie, celle où la jeune femme "voit des bouches ouvertes, ensanglantées, souiller encore une fois son sein nu ». Car cette image très forte sur laquelle se clôt le songe, Joseph apparemment ne la doit à personne : elle est de son cru. Elle relève bien sûr d'une esthétique qui lui est propre, cet expressionnisme baroque que lui a sans doute inspiré la lecture de la Thébaïde de Stace et qu'il pratique volontiers. Mais il ne la met pas en œuvre partout. Elle procède donc ici d'un choix raisonné, peut-être destiné à fixer à travers une image particulièrement frappante, empreinte à la fois de sexe, de souillure et de sang, les conséquences dommageables de l'action d'une " jeunesse " "vouée à la violence " qui constitue au XII ${ }^{e}$ siècle, "dans la société chevaleresque ", le principal "organe d'agression et de tumulte ", pour reprendre les termes employés par Georges Duby, et qui dans sa chasse frénétique aux héritières ne reculait pas toujours devant le rapt et le viol 45 . À travers le regard épouvanté d'une femme, le clerc qu'est Joseph, voué à Pallas, déesse de la sagesse, comme Henri III et Hector le sont à Bellone et à Mars, cherche sans doute à faire percevoir la sauvagerie bestiale qui constitue l'envers de toute action héroïque, et donc à donner une leçon de morale.

"S’il suffit d'un couple parfait pour vous faire admettre la guerre... ", disait l'Hélène de Giraudoux. Sans être diabolisé comme le couple formé par Hélène et Pâris,

42 L'Iliade, V, 455-456: "Heu, morimur, succurre, pater! "Vix principe iussus/ tandem heret.

43 Comme le note C. Croizy-Naquet, qui s'interroge à ce sujet ("Le portrait d'Hector", p. 74-75). Cf. Darès, De excidio, XII, 3.

44 Virgile, Énéide, X, 324 (prima lanugine) et 327 (iuvenum). Cf. Juvénal, Satires, XIII, 59 (où la prima lanugo s'oppose à la sacrae senectae, "l'auguste vieillesse ") et L'Iliade, IV, 5354 : faciem lanugo serenam/ induit (« le duvet de la jeunesse couvre son visage serein »). 
le couple d'Hector et d'Andromaque, apparemment uni dans une même perfection héroïque, mais souterrainement miné et finalement détruit par la violence guerrière, n'est pas, dans l'Iliade de Joseph d'Exeter, vraiment parfait. Le regard du clerc se fait même singulièrement pénétrant pour percevoir et dénoncer l'insuffisance d'un héroïsme guerrier purement profane qui fait de ses adeptes, comme l'écrit son contemporain Gautier Map, "de splendides palais du péché ». La relecture qu'il propose des personnages d'Andromaque et d'Hector fournit ainsi la preuve que son épopée n'est pas, malgré l'esthétisme raffiné qui s'en dégage, un simple exercice de style, et qu'il a réfléchi sur les modèles légués par la tradition antique pour en tirer des leçons adaptées aux problèmes que posaient les êtres et la société de son temps.

Francine MORA

Université de Versailles - Saint-Quentin-en-Yvelines

21 bis, rue Lamartine

91400 Orsay

Francine.Mora@poetiques.uvsq.fr 